\title{
1980: un écrivain romand est une femme
}

\section{Valérie Cossy}

\section{(2) OpenEdition}

Journals

Édition électronique

URL : http://journals.openedition.org/edl/2396

DOI : $10.4000 /$ edl.2396

ISSN : 2296-5084

\section{Éditeur}

Université de Lausanne

\section{Édition imprimée}

Date de publication : 15 mars 2020

Pagination : 135-140

ISBN : 978-2-940331-73-4

ISSN : 0014-2026

\section{Référence électronique}

Valérie Cossy, «1980: un écrivain romand est une femme », Études de lettres [En ligne], 312 | 2020, mis en ligne le 24 mars 2020, consulté le 17 septembre 2020. URL : http://journals.openedition.org/edl/ 2396 ; DOI : https://doi.org/10.4000/edl.2396 


\section{0: UN ÉCRIVAIN ROMAND EST UNE FEMME}

Avec l'attribution du Grand Prix C. F. Ramuz à Alice Rivaz le 29 novembre 1980, celle-ci rejoint un panthéon exclusivement masculin. Son nom s'inscrit à la suite de ceux qui, dès 1955, ont reçu ce prix prestigieux remis tous les cinq ans: Pierre-Louis Matthey, Charles-François Landry, Marcel Raymond, Philippe Jaccottet et Jacques Mercanton.

Au sein du jury de l'édition 1980, la ligne de démarcation entre les candidats et leurs défenseurs correspondait, au terme de la première réunion du comité, à un partage des eaux entre les hommes et les femmes: Jean-François Piguet, Jean-Paul Verdan et Jean Roudaut soutenant Robert Pinget d'un côté, alors que Doris Jakubec et Anne Perrier parvenaient à maintenir la candidature d'Alice Rivaz au point d'imposer, circonstance exceptionnelle dans l'histoire du prix, une seconde séance de délibération, au cours de laquelle bascula le vote. Le processus avait été d'autant plus long que, lors du premier tour de table, aucun des trois jurés masculins n'avait mentionné Alice Rivaz: sans Anne Perrier et Doris Jakubec, son nom serait demeuré ignoré au sein de ce qu'ellemême avait qualifié de "firmament noir du langage féminin" pour dénoncer, dès 1945, l'absence des femmes de la tradition littéraire.

Doris Jakubec, qui fut, dès 1981, directrice du Centre de recherches sur les lettres romandes (CRLR) et l'une des toutes premières femmes professeures à l'Université de Lausanne, était sensible aux risques d'incompréhension planant sur une pionnière. Quant à Anne Perrier, qui eut parfois l'impression douloureuse d'écrire dans l'ombre de Jaccottet, elle se reconnaissait dans la non-reconnaissance d'Alice Rivaz. Admirant l'intégralité de l'œuvre, elles avaient été particulièrement impressionnées par Jette ton pain (1979), livre-somme génial, susceptible de marquer le 


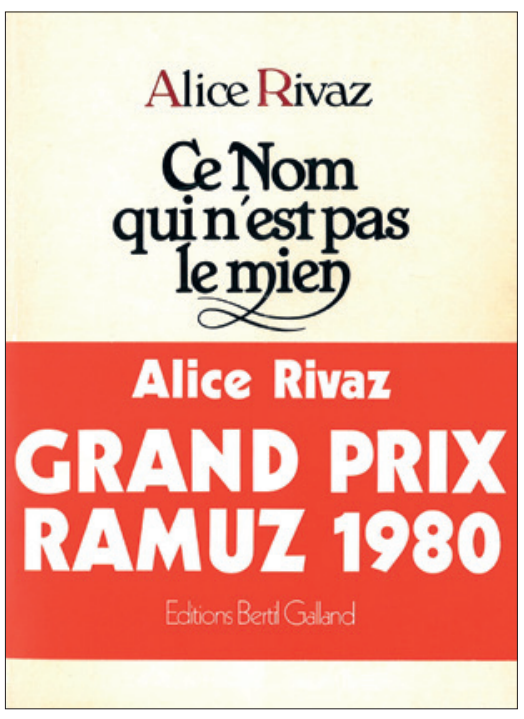

Grand Prix Ramuz en évoquant les contraintes matérielles et symboliques subies par les écrivaines. Ce dernier roman constituera le fil rouge de la laudatio de Doris Jakubec.

Si Gérard Buchet, au seuil de la cérémonie, introduit d'emblée Alice Rivaz comme la fille de Paul Golay - cette «haute personnalité politique et philosophique de notre pays»-, elle-même ne se fait pas faute de rappeler à l'assemblée la qualité fondamentale de son père: celle de grand démystificateur. Ainsi annonce-t-elle le plus sérieusement du monde qu'elle va citer son discours d'investiture comme doyen du Grand Conseil en 1949: «La charge que j'assume à cette heure, je la dois à mon âge et non à mes mérites. Je ne saurais donc susciter l'envie de personne.» S'appuyer sur l'exemple mordant de son père signifie résister à la tradition et conserver intact son esprit critique au moment même de la gloire, que l'on soit d'un parti minoritaire ou féministe. C'est aussi avertir son auditoire et ses lecteurs que son propre discours relève pour partie de cette ironie. Après tout n'a-t-elle pas dû atteindre l'âge de presque 80 ans pour que les tenants vaudois de la culture la prennent au sérieux?

Dans la suite de son discours, elle se présente comme une survivante, alors que "quatre femmes écrivains disparues" auraient tout autant qu'elle droit aux honneurs et, surtout, à la mémoire. Produisant son propre palmarès, elle annonce: «Je nomme ici Monique Saint-Hélier et Catherine Colomb qui toutes deux avaient du génie.» Elle évoque aussi Alice Curchod, "la plus méconnue de son vivant", et Corinna Bille, morte prématurément une année plus tôt, dont les contes et nouvelles relèvent de "cette littérature universelle capable de toucher tous les peuples». Passant sur quelques détails, la vieille dame n'a pas oublié de mentionner son travail d'anthologiste aux côtés de Ramuz, en 1940, alors que, tout en participant anonymement à la constitution de la tradition poétique, elle avait constaté en privé dans ses notes à propos des 
classiques: "Le Temps, certes, garde beaucoup de bon grain, mais pas tout le bon grain."

Doris Jakubec se souvient bien de la cérémonie. Elle qui n'avait jamais rencontré personnellement l'écrivaine fut surprise par ses manières de séductrice, un peu déroutantes de la part d'un "grand écrivain". Et, à part une petite verrée pour clôturer la cérémonie, il n'y eut pas de vrai moment festif ou de repas collectif pour célébrer la lauréate. Selon Doris Jakubec, «il restait l'ombre de Pinget». Parmi tous les services rendus aux femmes, Alice Rivaz aura donc affronté ce jour-là le préjugé inséparable des "premières fois": passer aux yeux de certains pour celle qui prend la place d'un homme. Or son habitus, c'est un fait, n'était pas celui d'un homme de lettres, comme elle s'en est souvent amusée dans ses écrits.

Mais Alice Rivaz savait bien que s'envolent les paroles et restent les écrits. Aux côtés de son éditeur Bertil Galland, dont son discours salue "le dévouement inlassable et l'amitié agissante», elle publie à cette occasion Ce nom qui n'est pas le mien. Sachant qu'une femme de sa génération n'est jamais mieux servie que par elle-même, elle travaille à sa mémoire. Consulté sur la composition de ce recueil, Bertil Galland précise: "C’est Alice Rivaz, elle seule, qui a composé le manuscrit de Ce nom qui n'est pas le mien. Elle n'était pas seulement grande en littérature par son écriture, mais aussi, sans qu'elle s'en prévale, une professionnelle du travail d'écrivain et de l'édition ». Comme Virginia Woolf dont elle admirait la «liberté totale», elle maîtrise ici toutes les étapes de la création littéraire.

Il faut souligner notamment, dans Ce nom qui n'est pas le mien, la reprise d'un texte de 1945: "Un peuple immense et neuf» peut être considéré comme le premier texte de critique littéraire féministe en français. Avec trente ans d'avance au calendrier, la marginale Alice Rivaz était en phase avec la génération féministe post-Beauvoir de 1980. Dans son invitation à tout déconstruire, Hélène Cixous avait ainsi lancé son pavé dans la mare en 1975: «Presque toute l'histoire de l'écriture se confond avec l'histoire de la raison [...]. Elle a été homogène à la tradition phallocentrique.» Dans son essai de 1945, Alice Rivaz avait ellemême décrit l'écrivaine aliénée comme «inévitablement prête à laisser sa féminité à côté de sa bouteille d'encre et à tremper sa plume dans celle de la vénérable culture masculine», une formule reprise quasiment mot pour mot dans son discours du Grand Prix Ramuz.

Mais, passé l'événement, la question féministe incontournable dans le monde social devient un refoulé de la critique littéraire en Suisse 
romande comme en France. Ayant droit de cité en sociologie, le féminisme francophone a été tenu à distance de toute interrogation fondamentale sur la production symbolique: seules une Hélène Cixous ou une Monique Wittig se sont risquées à franchir le mur idéaliste de la critique littéraire en français, devenant immédiatement des voix de référence célébrées en tant que telles... dans le monde anglophone. Le cas d'Alice Rivaz permet donc de réfléchir à la nature contrariée de la rupture féministe au sein de la littérature d'expression française. Si 1980 inaugure une reconnaissance des écrivaines et de leurs interrogations par le milieu de la création littéraire, l'on ne saurait en dire autant, hélas, de la critique universitaire. La Faculté des lettres de l’Université de Lausanne, il faut l'admettre, est encore à l'image de ce qu'Alice Rivaz dénonçait ironiquement il y a quarante ans lorsqu'elle disait «bénéficier ", à près de 80 ans, «du retard avec lequel est reconnu dans l'ensemble des pays francophones, contrairement à ce qui s'est passé dans les pays anglo-saxons, l'existence et le rayonnement d'une littérature féminine». Chez nous, en 2020, les études femmes, la critique féministe et leurs développements ultérieurs - le genre et le queer - sont toujours exilés en section d'anglais comme un exotisme de la pensée.

Valérie Cossy

Section d'anglais, Faculté des lettres, Université de Lausanne 


\section{BIBLIOGRAPHIE}

Entretien et courriels avec Doris Jakubec et Bertil Galland, juin 2019.

Bulletin de la Fondation C. F. Ramuz, Pully, 1981.

Cixous, Hélène, Le rire de la Méduse et autres ironies, Paris, Galilée, 2010.

Galland, Bertil, Une aventure appelée littérature romande, Genève, Slatkine, 2014.

Golay, Paul, Terre de justice, Lausanne, Imprimeries populaires, 1951.

Ramuz, Charles Ferdinand, Anthologie de la poésie française, Paris, Corrêa, 1943.

Rivaz, Alice, Ce nom qui n'est pas le mien, Vevey, Éditions Bertil Galland, 1980.

—, Traces de vie, Vevey, Éditions Bertil Galland, 1983.

Rivaz, Alice, Girard, Pierre, Les enveloppes bleues, Correspondance 1944-1951, sous la dir. de Daniel Maggetti, Carouge-Genève, Zoé, 2005.

Wittig, Monique, The Straight Mind and Other Essays, Boston, Beacon Press, 1992 ( $1^{\text {re }}$ édition française: La pensée straight, Paris, Balland, 2001). 
